\title{
The screening of heart disease by cardiac auscultation and electrocardiography examination in pregnant women in Puskesmas Tegalrejo, Yogyakarta, Indonesia
}

Lucia Kris Dinarti, ${ }^{1,3}$ Detty Siti Nurdiati, ${ }^{2,3}$ Anggoro Budi Hartopo, ${ }^{1,3}$ Fika Humaeda Assilmi, ${ }^{1,3}$ Alifia Salsabila, ${ }^{1}$ Vera Dewanto, ${ }^{1}$ Armalya Pritazahra, ${ }^{1,3}$ Muhammad Reyhan Hadwiono, ${ }^{1}$ Muhammad Gahan Satwiko, ${ }^{1,3}$ Erna Ashlihah Rochmat, ${ }^{2,3}$ Pravitasari, ${ }^{2,3}$ Dyah Wulan Anggrahini ${ }^{1,3, *}$

${ }^{1}$ Department of Cardiology and Vascular Medicine, Faculty of Medicine, Public Health, and Nursing, Universitas Gadjah Mada, Yogyakarta, Indonesia

${ }^{2}$ Department of Obstetrics and Gynecology, Faculty of Medicine, Public Health, and Nursing, Universitas Gadjah Mada, Yogyakarta, Indonesia

${ }^{3}$ Dr Sardjito General Hospital, Yogyakarta, Indonesia

\section{KEYWORDS}

Cardiovascular diseases

Electrocardiograph

Screening

Pregnancy
ABSTRACT Women adapt to pregnancy through multi-organ system physiologic changes, including cardiovascular adaptations. These changes affect those with pre-existing cardiovascular problems differently, and subsequently lead to higher probability of death caused by cardiovascular diseases during pregnancy. Therefore, detection of cardiovascular disease early in pregnancy is important to lower maternal morbidity and mortality by providing prompt and adequate management. This study aimed to evaluate and test the feasibility of integrating 12-lead electrocardiogram (ECG) examination and antenatal care (ANC) screening as a simple and effective method for early detection of heart abnormality in pregnant woman. Pregnant women were recruited in this study in any trimester who attended ANC for a routine pregnancy examination in Puskesmas Tegalrejo Yogyakarta. The subjects underwent primary screening which focused on cardiac auscultation and 12-lead ECG examinations, and those who had abnormal findings were further followed-up in secondary screening by using trans-thoracic echocardiography to confirm heart abnormality. A total of 523 pregnant women from Puskesmas Tegalrejo were included in this study. 15 (2.8\%) pregnant woman were suspected to have heart abnormalities; from those, $3(0.5 \%)$ were found with heart murmurs with abnormal ECG readings, $1(0.19 \%)$ had heart murmurs with normal ECG results, and $11(2.1 \%)$ had abnormal ECG readings only. The secondary screening of those patients resulted in $1(0.19 \%)$ pregnant woman who was diagnosed with Atrial Septal Defect. Our study found that among 15 patients identified with suspected ECG abnormalities, one mother who underwent ANC was newly diagnosed with a pre-existing cardiac abnormality. Our study concluded this screening method is a simple and feasible integrated heart screening program that can be implemented widely. We hope this integrated heart screening program may benefit pregnant women who may have cardiac abnormalities to be detected as early as possible, thus reducing maternal morbidity and mortality.

(c) The Journal 2021. This article is distributed under a Creative Commons Attribution-ShareAlike 4.0 International license.

\section{Introduction}

Globally, the maternal mortality is considerably high. Based on the World Health Organization (WHO) data in 2017, approximately 295,000 women died during and following pregnancy and labor. Almost all of these deaths (94\%) happened in low-resource settings and most of the cases were preventable. To battle the rising number of maternal mortalities, as well as other

\footnotetext{
*Correspondence: wulan.anggrahini@ugm.ac.id

Department of Cardiology and Vascular Medicine, Faculty of Medicine, Public Health, and Nursing, Universitas Gadjah Mada, Jl. Farmako, Sekip Utara, Yogyakarta 55281, Indonesia
}

concerning problems in the world, world leaders have agreed to propose certain public health target goals to reach. The Millennium Development Goals (MDGs) target which was needed to be achieved for maternal mortality rate was 102 per 100,000 live birth rates by 2015 . However, existing data showed that in Indonesia by 2015, maternal mortality rate was 305 per 100,000 live birth rate, which was three times higher than the MDGs target. ${ }^{1}$ In 2015, at the United Nations (UN) Sustainable Development Summit, a new agenda was adopted in the form of 17 Sustainable Development Goals (SDGs). One of the goals is to reduce the global maternal mortality 
ratio to less than 70 per 100,000 live births by 2030 . All UN member states, including Indonesia, have pledged to implement these SDGs. Accordingly, Indonesia has been working hard to tackle causes of maternal mortality by conducting various regulations and health promotions. ${ }^{2}$

In pregnancy, various physiological changes occur in blood volume, red cell mass, left ventricular mass and elevated cardiac output (CO) which will increase cardiovascular demands to meet the needs of the fetus. ${ }^{3}$ Mortality and morbidity of pregnant women who have a pre-existing cardiac abnormality or develop cardiac problems during pregnancy are increasing worldwide. ${ }^{4}$

Cardiovascular disease (CVD) is one of the causes of death during pregnancy in developed countries, and cardiovascular complications may develop in $0.2 \%-4 \%$ of pregnancies, even if no prior cardiac disease was detected. ${ }^{4,5}$ In another study, one of the diseases which was commonly found in pregnancy is pulmonary arterial hypertension (PAH). ${ }^{2}$ Congenital Heart Disease (CHD) is associated and included in the PAH subgroup. ${ }^{2}$ Data showed that women are affected 3-4 times more frequently than men. ${ }^{2}$ Pregnant women with PAH were reported to have high rates of maternal (30-56\%) and fetal death (11$28 \%$ ). Progressive pulmonary vascular obliteration can cause right heart failure and stroke from intracardiac shunting, which are examples of various preventable causes of poor maternal outcomes. ${ }^{7}$

Data from the Indonesian Ministry of Health in 2015 showed that the maternal mortality rate is 305 out of 100,000 births, and the maternal mortality rate due to heart disease in pregnancy ranges from $1-2 \%$. The risk of maternal death is increased in cases with pulmonary hypertension, Eisenmenger syndrome, coarctio aorta, and complicated Marfan syndrome. ${ }^{8}$ Data acquired from Indonesia Ministry of Health in 2017 stated that maternal mortality in Yogyakarta in the year of 2017 were 34 per 42,348 live births $(0.08 \%)$. The causes of death were mostly due to heart disease (29\%), with $0.29 \%$ embolism, $14 \%$ bleeding, $0.29 \%$ eclampsia, and $0.9 \%$ caused by pre-eclampsia. ${ }^{1}$ The COHART PH registry from a single center study in Indonesia showed that $\mathbf{7 8 0}$ (77\%) of 1,012 patients were females who were first diagnosed at reproductive age ranging in age between $18-40$ years old and $67 \%$ have had PAH. ${ }^{9}$

One of the programs in Indonesia that was developed to reduce the high maternal mortality rate in Indonesia is the optimization of Antenatal Care (ANC). ANC is performed routinely during scheduled visits for pregnant women and focuses on general physical examinations and obstetric examinations. Specific heart or cardiovascular examinations are rarely performed, thus patients with asymptomatic CVD could be undetected. Several studies on cardiovascular abnormality screening methods have been performed by combining cardiac auscultation, 12 lead electrocardiogram (ECG) and echocardiography.,10 The ECG examination is a simple, effective, and easy to perform method for diagnosing CVD. Based on our previous study, this program has been proven effective as a screening method of CVD in children. ${ }^{10}$ However, in Indonesia this examination has never been performed routinely in pregnant women. Thus, the main purpose of this study was to evaluate and assess the ability of this ANC screening program by using cardiac auscultation and 12-lead ECG to detect any heart abnormality in pregnant women.

\section{Method}

An observational-descriptive study was performed to analyze the application of cardiac abnormality screening methods based on physical examination and ECG among pregnant women in Yogyakarta. The screening program was conducted by a team of professional health providers in Puskesmas Tegalrejo, Yogyakarta. This screening program was integrated with the routine ANC visits in the Puskesmas.

Purposive sampling was performed to enroll pregnant women who had ANC in Puskesmas Tegalrejo in Yogyakarta. Verbal and written consent were collected from all participants. This screening method was integrated with ANC services in Puskesmas Tegalrejo and consisted of two stages, primary and secondary screening. Primary screening was performed between May 2019 - October 2019 and secondary screening was performed in November 2019.

Primary screening examination utilized physical 


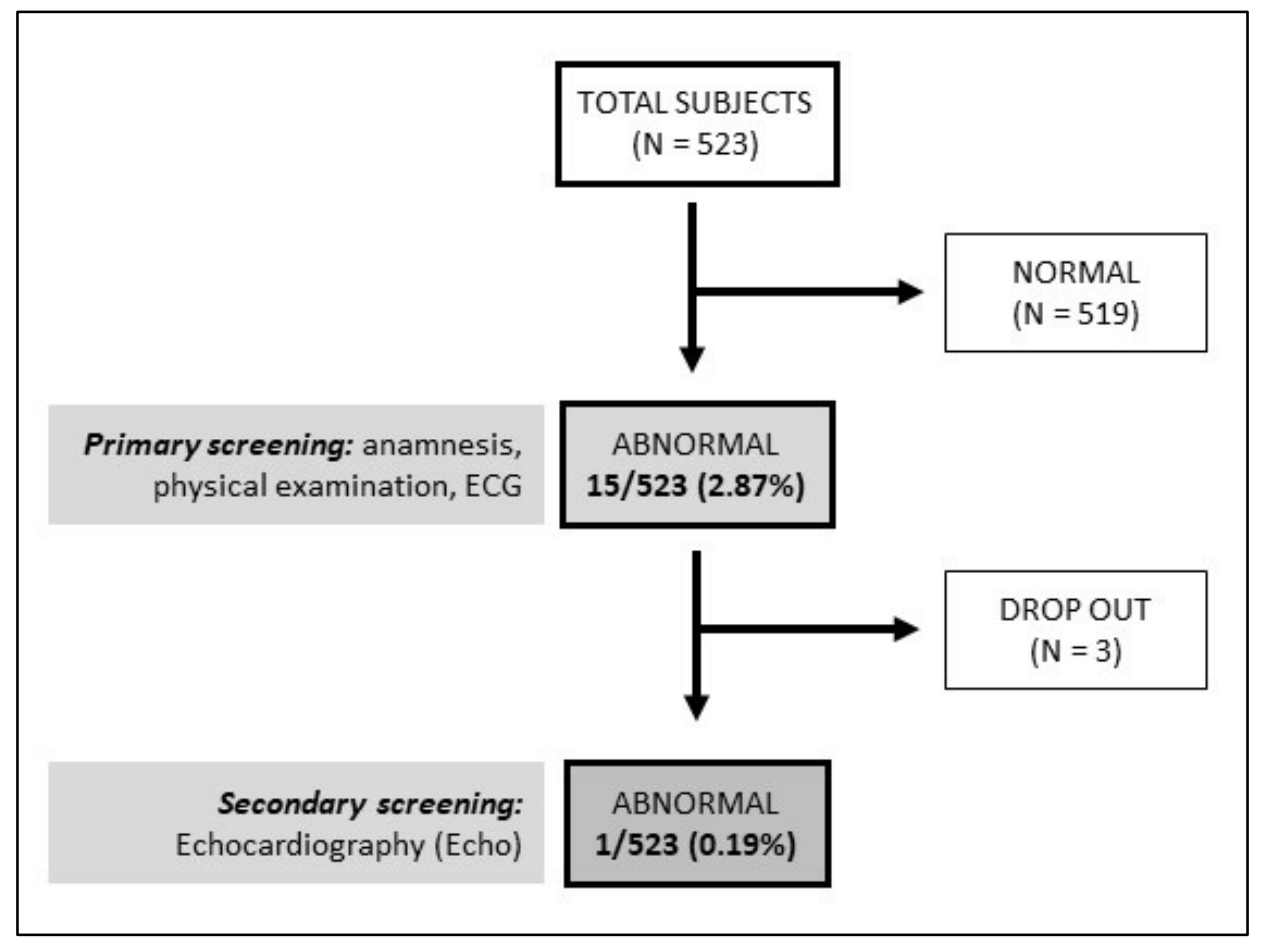

Figure 1. Study flowchart of integrated cardiac screening in ANC.

examinations (inspection, percussion, palpation, and auscultation using cardiology stethoscope) and 12-lead ECG examination. The 12-lead ECG was made up of three standard limb leads (I, II, III), three augmented limb leads (aVR, aVL and aVF) and six precordial leads (V1, V2, V3, V4, V5, V6). Secondary screening was given to pregnant women who showed an abnormal ECG reading from primary screening and/or had abnormal heart murmurs. During the secondary screening, to find and diagnose any heart abnormality transthoracic echocardiography was used.

The 12-lead ECG and physical heart examinations were performed bygeneral practitionersand mid wives from Puskesmas Tegalrejo who previously had been trained to use standard methods. Interpretations of the ECG reading were categorized into normal and abnormal. Cardiologists then confirmed the abnormal ECG readings that had been found. The suspected pregnant woman with abnormal heart murmurs and/or confirmed abnormal ECG readings would undergo secondary screening by a trained cardiologist.

Data analysis was performed using SPSS Statistics Version 22 (IBM Corp., Armonk, NY). Data were presented as means and standard deviations (SD) or median and interquartile ranges or proportions, as appropriate.

\section{Result}

A total of 523 pregnant women of any trimester from one primary health care center were participated in the screening and follow the flow of the screening algorithm (Figure 1). The mean of age was 28.59 $( \pm 5.75)$ years old and most of the subjects were multiparous, which was 312 (61.56\%) pregnant women. The characteristics of the participants are shown in Table 1. From 523 participants, we found $15(2.8 \%)$ pregnant woman were suspected to have heart abnormalities. Based on the results of primary screening, $3(0.5 \%)$ women were found with heart murmurs with abnormal ECG readings, 1 (0.19\%) had heart murmurs with normal ECG results, and 11 (2.1\%) had abnormal ECG readings only. There are several ECG abnormalities and 2 types of heart sound anomalies observed among the subjects as shown in Figure 2 . The secondary screening of those patients resulted in 1 (0.19\%) pregnant woman was diagnosed with Atrial Septal Defect. Table 2 shows the findings of the primary and secondary screenings. 
Table 1. Baseline characteristics of subjects

\begin{tabular}{lll}
\hline & $\mathrm{N}(\%)$ & Mean $( \pm$ SD) \\
\hline Age (years old) & & \\
Parity & & \\
$\quad$ Multiparous & $312(61.59 \%)$ & \\
$\quad$ Nulliparous & $211(40.35)$ \\
Heart Rate (x/min) & & \\
Oxygen saturation (\%) & & $83.86( \pm 10.05)$ \\
\hline
\end{tabular}

Table 2. Incidence of cardiac abnormality during ANC

\begin{tabular}{ll}
\hline Screening & Incidence \\
\hline Primary screening & $15 / 523(2.87 \%)$ \\
Heart murmurs with abnormal ECG readings & $3(0.57 \%)$ \\
Heart murmurs with normal ECG readings & $1(0.19 \%)$ \\
Abnormal ECG readings only & $11(2.10 \%)$ \\
\hline Secondary screening & $1 / 523(0.19 \%)$ \\
Congenital Heart Disease (Atrial Septal Defect) & $1(0.19 \%)$ \\
\hline
\end{tabular}

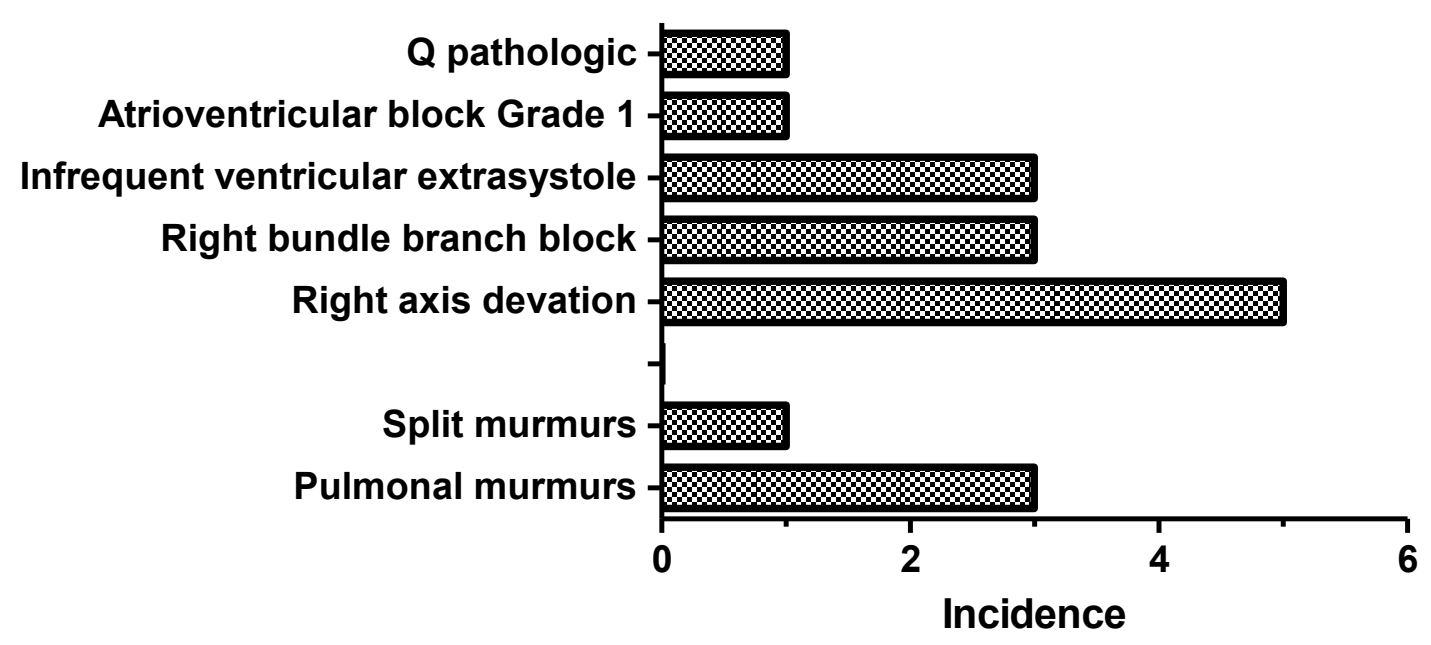

Figure 2. Types of abnormal findings from primary screening

\section{Discussion}

The screening program of heart disease in pregnant women by using 12-lead ECG was the first systematic program that focused on heart abnormality in pregnant women in Indonesia. With collaboration between midwives, general practitioners, cardiologists and obstetricians, this program ran well and provided satisfactory results. This study found several patients with newly diagnosed cardiac abnormality among 523 subjects who attended routine ANC, which included 1 (0.19\%) participant with previously undiagnosed CHD.

Cardiovascular diseases (CVD) are the leading cause of maternal death in the world. The variability of CVD rates and diagnostic criteria among pregnant women are different between countries according to European Society of Cardiology (ESC) and American College of Cardiology/American Heart Association (ACC/AHA) guidelines. ${ }^{6}$ In the Western countries, $\mathrm{CHD}$ is the most common CVD during pregnancy (75\%-82\%), whereas in non-Western countries, rheumatic heart disease (RHD) is the most common 
CVD (56\%-89\%). ${ }^{5}$ A study by Subbaiah et al. described that cardiac disease was found to complicate $3.8 \%$ of pregnancies. From their retrospective study, most pregnant women were diagnosed with CVD in their third trimester. ${ }^{11}$ Common complaints found in pregnancy could mimic symptoms in CVD, such as shortness of breath and heart palpitations. These symptoms commonly occur during the third semester of pregnancy, which could also result from preexisting CVD. These are some of the many reasons for delays in diagnosing CVD in pregnancy, which subsequently can lead to inaccurate management, resulting in high maternal morbidity and mortality caused by CVD. ${ }^{4}$

In developed countries, several methods have been developed to detect and manage CVD during pregnancy. In the United States, one of the many efforts to combat this problem is a toolkit that includes an overview of clinical assessment and management strategies for CVD based on risk factors and presenting signs and symptoms. The toolkit recommends an algorithm for clinicians to use as a guide to differentiate signs and symptoms related to cardiac diseases and those of normal pregnancy, as well as further follow-up. The algorithm is based on risk factors, symptoms, vital sign abnormalities, and physical examination findings commonly identified in women who die of various types of cardiovascular disease. One study was conducted to assess the toolkit and included 64 CVD induced maternal deaths. The study found that the use of the algorithm could identify 56 out of 64 (88\%) cases of CVD. ${ }^{12}$ However, this algorithm primarily detects serious cases of CVD disease in late stage pregnancy.

The rate of CVD in pregnant women has also increased in developing countries. Many factors have contributed to this transition, and the most prevalent reasons are due to the increased number of women with CHD reaching childbearing age and the demographic changes associated with advancing maternal age resulting from improvements in health care services. A study in Turkey found the CVD rate of 5.2\% among healthy women in the first trimester of pregnancy. The study enrolled pregnant women in their first trimester, without cardiac symptoms and previously known CVD history. All patients underwent physical cardiac examinations, 12-lead
ECG, and transthoracic echocardiography. The study suggested that based on their findings, all pregnant women who do not have any clinical risk or cardiac symptom, should be referred to a cardiologist and undergo ECG examination in their first trimester. Even though the cardiac screening would require additional funding, it could be lifesaving and costeffective since it would provide early recognition of pre-existing CVD that may become aggravated in pregnancy. ${ }^{7}$

Several risk scores are available as an important step in recognizing the risk of maternal complications during and after pregnancy in patients with CVD, and one of those is the modified WHO (mWHO) risk classification. The stratification is based on the underlying diagnoses, therefore previous history of CVD should be known. Investigation before pregnancy should be done consisting of a thorough history taking, physical examinations, an ECG, echocardiogram and exercise test. Complete physical examination is required to recognize heart murmurs, gallop sound, elevated central venous pressure, rales, hepatosplenomegaly and peripheral edema. An ECG could provide prognostic information, for example, if a new or known atrial fibrillation is found. ${ }^{13}$ We adopted two simple examinations for this study's ANC screening, cardiac auscultation and 12-lead ECG.

Due to the high mortality rate, the Indonesian Government has established a proposal to reduce maternal mortality in the 2014-2019 National Medium-Term Development Plan (RPJMN). One of the programs is the ANC. Indicators used to evaluate the access of pregnant women to ANC services are $\mathrm{K} 1$, the first contact in the first trimester and K4, 4 contacts in the final trimester. Nationally, the rate of coverage for ANC services is considerably high, with K1 reaching $92.24 \%$ and K4 reaching $84.36 \%$. Nevertheless, the current ANC program focuses on physical examinations and supporting examinations related to pregnancy problems, which have limitations in detecting heart disease in pregnant women. ${ }^{1}$ We integrated our heart screening program into the current ANC, in order to save time and resources, as well as to provide a more comprehensive care.

These findings highlight the feasibility and ability to perform and integrate the heart screening for 
pregnant women within the current ANC program by using cardiac auscultation and 12-lead ECG. The team from Puskesmas Tegalrejo with the help of our team have successfully performed hundreds of examinations of primary screening. Training was conducted to refresh the technique to perform cardiac auscultation and 12-lead ECG and to recognize heart murmurs and normal patterns of ECG reading. Several limitations were observed regarding the cardiac screening. The ANC service is provided during working hours of Puskesmas, thus it runs parallel with the general polyclinics. The shortage of general practitioners and available ECGs at the Puskesmas were the main problems of the program. Another limitation of this study is the possibility of bias and subjectivity of cardiac auscultation by primary health clinicians or general practitioners during the primary screening. Trainings on heart auscultation prior to primary screening for primary health cares' doctor are needed to improve their performance. Nevertheless, this primary screening approach was well-received by the participants and considerably uncomplicated and easy to perform.

\section{Conclusions}

Cardiovascular screening during pregnancy could use simple tools that are effective, not expensive, and available even in rural areas in Indonesia. Early detection of cardiac diseases during pregnancy can be lifesaving and cost-effective because cardiac problems continue to be one of the major causes of maternal deaths worldwide including Indonesia.

Based on our study, among several patients detected with a suspected cardiac abnormality, there was one mother identified with a previously undiagnosed CHD ( $n=1,0.19 \%)$ in 523 healthy pregnant women in any trimester. This study showed that it is possible to integrate heart screening with ANC for pregnant women in Yogyakarta city by using cardiac auscultation and 12-lead ECG. However, logistics such as ECG availability as well as human resources are important considerations that need to be prepared for the primary health care center or by the government to integrate heart disease screening with ANC so that the program can be optimally implemented. Another important factor that needs to be done is proposing a standard algorithm for routine cardiac screening to diagnose cardiac abnormality early in pregnancy, to achieve the maximum benefits.

\section{Acknowledgement}

Authors acknowledge the facilitation and support of Puskesmas Tegalrejo. Authors deeply appreciate the contributions of general practitioners and midwives from Puskesmas Tegalrejo. Authors acknowledged the assistance Cardiology Residents in the execution of this program. This study was supported by Hibah DAMAS Penelitian 2019 Faculty of Medicine, Public Health, and Nursing Universitas Gadjah Mada, Yogyakarta Indonesia for Lucia Kris Dinarti as principal investigator.

\section{Conflict of interests}

None.

\section{References}

1. Dinas Kesehatan Daerah Istimewa Yogyakarta. Profil Kesehatan Daerah Istimewa Yogyakarta Tahun 2019. Yogyakarta. 2020; 21-31.

2. Sustainable Development Goals [Internet]. Who. int. 2021 [cited 1 January 2021]. Available from: https://www.who.int/health-topics/sustainabledevelopment-goals\#tab=tab_1

3. Hemnes A, Kiely D, Cockrill B, Safdar Z, Wilson $V$, Hazmi $M$ et al. Statement on pregnancy in pulmonary hypertension from the Pulmonary Vascular Research Institute. Pulmonary Circulation. 2015;5(3):435-465.

4. Adam K. Pregnancy in women with cardiovascular diseases. Methodist Debakey Cardiovasc J. 2017;13(4):209-15.

5. Bouvier-Colle $M H$, Mohangoo $A D$, Gissler $M$, Novak-Antolic Z, Vutuc C, Szamotulska K, et al. What about the mothers?: an analysis of maternal mortality and morbidity in perinatal health surveillance systems in Europe. BJOG An Int J Obstet Gynaecol. 2012;119(7):880-90.

6. Gordon CA, Rosenthal AH, O'Leary JL. Heart disease and pregnancy. Am J Surg. 1952;83(1):7282.

7. Bozkaya V, Kaplan Z, Özgü E, Engin-Üstün Y. The 
screening and evaluation of newly diagnosed cardiovascular diseases in first trimester asymptomatic pregnant women in a tertiary antenatal care center in Turkey. Anatol J Cardiol. 2019 Jan;23(2):99-104.

8. Seeland U, Bauersachs J, Roos-Hesselink J, RegitzZagrosek V. Update of the ESC guidelines 2018 on cardiovascular diseases during pregnancy: most important facts. Herz. 2018;43(8):710-8.

9. Dinarti $L K$, Hartopo $A B$, Kusuma $A D$, Satwiko MG, Hadwiono MR, Pradana $A D$, et al. The COngenital HeARt Disease in adult and Pulmonary Hypertension (COHARD-PH) registry: a descriptive study from single-center hospital registry of adult congenital heart disease and pulmonary hypertension in Indonesia. BMC Cardiovasc Disord. 2020;20(1):1-11.
10. Dinarti LK, Murni IK, Anggrahini DW, Dewanto VC, Pritazahra A, Hadwiono MR, et al. The screening of congenital heart disease by cardiac auscultation and 12-lead electrocardiogram among Indonesian elementary school students. Cardiol Young. 2020;1-10.

11. Subbaiah M, Sharma V, Kumar S, Rajeshwari $\mathrm{S}$, Kothari SS, Roy KK, et al. Heart disease in pregnancy: cardiac and obstetric outcomes. Arch Gynecol Obstet. 2013;288(1):23-7.

12. Hameed $\mathrm{AB}$, Morton $\mathrm{CH}$, Moore $\mathrm{A}$. Improving health care response to cardiovascular disease in pregnancy and postpartum. California Maternal Quality Care Collaborative. 2017;11.

13. Van Hagen IM, Roos-Hesselink JW. Pregnancy in congenital heart disease: risk prediction and counselling. Heart. 2020;106(23):1853-61. 